\title{
Tobacco smoking and semen quality in infertile males: a systematic review and meta-analysis
}

\author{
Pravesh Kumar Bundhun', Girish Janoo², Akash Bhurtu², Abhishek Rishikesh Teeluck², \\ Mohammad Zafooruddin Sani Soogund ${ }^{2}$, Manish Pursun ${ }^{2}$ and Feng Huang ${ }^{3^{*}}$ (i)
}

\begin{abstract}
Background: Nowadays, the total number of couples visiting an infertility clinic is on the rise. Tobacco smoking is considered one of the major factors leading to male infertility. In this study, we aimed to systematically investigate the impact of tobacco smoking on semen quality in infertile male participants.

Methods: Online databases (Cochrane Central database of Randomized Controlled Trials and the databases of MEDLINE and EMBASE respectively) were searched for relevant English publications that satisfied the inclusion and exclusion criteria of this analysis. The clinical endpoints which were assessed included semen parameters (oligozoospermia, asthenozoospermia, teratozoospermia, and azoospermia), morphological defects of spermatozoa and the hormones involved in reproduction. RevMan 5.3 software was used to analyze the data whereby mean difference (MD) and risk ratios (RR) with 95\% confidence intervals (Cl) were generated to represent the results.

Results: Sixteen studies with a total number of 10,823 infertile male participants (5257 smokers and 5566 non-smokers) were included. Results of this analysis showed oligozoospermia to be significantly higher in smokers (RR: 1.29, 95\% Cl: 1.05$1.59 ; P=0.02$ ). Morphological defect of spermatozoa (MD: $2.44,95 \% \mathrm{Cl}: 0.99-3.89 ; P=0.001$ ) was also significantly higher in smokers whereby significant head (MD: 1.76, 95\% Cl: 0.32-3.20; $P=0.02$ ), neck (MD: 1.97, 95\% Cl: 0.75-3.18; $P=0.002$ ) and tail (MD: 1.29, 95\% Cl: 0.35-2.22; $P=0.007$ ) defects were observed. However, smoking did not affected the pH (MD: 0.04, 95\% Cl: [ $-0.03-0.11] ; P=0.30$ ) and motility (RR: $1.42,95 \%$ Cl: $0.97-2.09 ; P=0.07)$ of spermatozoa. Additionally, tobacco smoking did not cause any dis-balance in hormones which were involved in reproduction.

Conclusions: In conclusion, with reference to the clinical endpoints which were studied in this analysis, tobacco smoking was associated with a lower sperm count and an increase in the number of morphological defects of spermatozoa. However, the pH and motility of spermatozoa as well as the production of hormones which were involved in reproduction were not affected in this population of infertile males.
\end{abstract}

Keywords: Smoking, Infertile men, Semen, Oligozoospermia, Asthenozoospermia, Teratozoospermia, Azoospermia

\section{Background}

Tobacco smoking among the young generation is becoming worse day by day [1]. The effect of tobacco smoking on lung cancer is already well-known [2]. However, other serious health hazards of smoking have not often well

\footnotetext{
* Correspondence: huangfeng7925@163.com

${ }^{3}$ Institute of Cardiovascular Diseases and Guangxi Key Laboratory Base of Precision Medicine in Cardio-cerebrovascular Disease Control and Prevention and Guangxi Clinical Research Center for Cardio-cerebrovascular Diseases, the First Affiliated Hospital of Guangxi Medical University, Nanning 530021, Guangxi, China

Full list of author information is available at the end of the article
}

been investigated [3]. Not lately, there has been evidence showing tobacco smoking to have shocking impact on reproductive health irrespective of gender status.

Nowadays, the total number of couples visiting an infertility clinic is on the rise [4]. According to the American Society for Reproductive Medicine, infertility is defined as the inability to achieve pregnancy after a duration period of one year of regular, unprotected sexual intercourse [5].

Tobacco smoking is considered one of the major factors leading to male infertility [6] and recent surveys have demonstrated approximately 120,000 young men

(c) The Author(s). 2019 Open Access This article is distributed under the terms of the Creative Commons Attribution 4.0 International License (http://creativecommons.org/licenses/by/4.0/), which permits unrestricted use, distribution, and reproduction in any medium, provided you give appropriate credit to the original author(s) and the source, provide a link to the Creative Commons license, and indicate if changes were made. The Creative Commons Public Domain Dedication waiver (http://creativecommons.org/publicdomain/zero/1.0/) applies to the data made available in this article, unless otherwise stated. 
(30 to 50 years old) in the United Kingdom to be impotent due to this bad habit. Male infertility (approximately $50 \%$ of the cases of infertility among couples [7]) is gradually leading to depression and other psychological outcomes, and this might be potential signs of serious future consequences.

The impact of tobacco smoking on semen quality has seldom been systematically studied. Therefore, by comparing semen parameters between smokers and non-smokers, we aimed to systematically investigate the impact of tobacco smoking on semen quality in infertile male participants.

\section{Methods}

\section{Electronic databases and searched strategies}

An electronic search was carried out for English language publications through the Cochrane Central database of Randomized Controlled Trials, the databases of MEDLINE (Medical-related publications) and EMBASE respectively. The terms 'smoking and infertility', 'smoking and male infertility', 'smoking and semen', 'smoking and sperm,' 'smoking and young males', 'infertility and tobacco smoking,' smoking and male health', 'smoking, males and impotence,' 'smoking and infertile men', 'smoking and sperm morphology', 'smoking and sex', smoking and sperm count,' 'smoking and testosterone, 'smoking and LH,' 'smoking and FSH', 'smoking and prolactin' and 'smoking and sperm motility' were used to find relevant publications.

To improve this search process, the terms 'males, men, cigarettes, nicotine, tobacco and non-fertile' were also included one at a time during the search process. In addition, reference lists of suitable articles were also reviewed for relevant publications.

\section{Inclusion criteria}

Inclusion criteria were based on the following features:

(a) Studies based strictly on infertile male participants;

(b) Studies that compared respective semen parameters in smokers versus non-smokers;

(c) Studies that reported the following endpoints: semen parameters, $\mathrm{pH}$ of semen, morphological defects of spermatozoa, types of abnormal structural defects, and hormones which were involved in the functioning of the male reproductive system.

\section{Exclusion criteria}

Exclusion criteria were based on the following features:

(a) Studies that consisted of fertile/normal male participants;

(b) Studies that did not compare respective semen parameters in smokers versus non-smokers; (c) Studies that did not report the above-mentioned endpoints;

(d) Duplicated studies.

\section{Endpoints}

Selective endpoints included:

- Oligozoospermia;

- Asthenozoospermia;

- Teratozoospermia;

- Azoospermia;

- Morphological defects of spermatozoa: head, neck or tail defects;

- pH of semen;

- Testosterone level;

- Follicle stimulating hormone (FSH) level;

- Luteinizing hormone (LH) level;

- Prolactin level.

The endpoints have been listed in Table 1.

Table 1 Reported endpoints

\begin{tabular}{|c|c|}
\hline Study & Selective endpoints reported \\
\hline Al-Turki2014 ${ }^{10}$ & $\begin{array}{l}\text { pH of semen, testosterone level, FSH level, LH level, } \\
\text { prolactin level }\end{array}$ \\
\hline Al-Turki2016 ${ }^{11}$ & Serum testosterone, semen $\mathrm{pH}$ \\
\hline $\begin{array}{l}\text { Anifandis } 2014 \\
12\end{array}$ & Sperm immotility \\
\hline Caserta2012 ${ }^{13}$ & $\begin{array}{l}\text { Oligozoospermia, asthenozoospermia, teratozoospermia, } \\
\text { FSH level, LH level }\end{array}$ \\
\hline Cui2016 ${ }^{14}$ & $\begin{array}{l}\text { Abnormal sperm head, abnormal sperm body, } \\
\text { abnormal sperm tail }\end{array}$ \\
\hline Gaur2007 ${ }^{15}$ & Oligozoospermia, asthenozoospermia, teratozoospermia \\
\hline Meri2013 ${ }^{16}$ & Serum $\mathrm{pH}$ \\
\hline Mitra2012 ${ }^{17}$ & $\begin{array}{l}\text { Asthenozoospermia (reduced motility), oligozoospermia } \\
\text { (low sperm count), teratozoospermia (sperm with } \\
\text { abnormal morphology), azoospermia (no sperm count), } \\
\text { immotility, sperm head defect, sperm tail defect }\end{array}$ \\
\hline $\begin{array}{l}\text { Trummer2002 } \\
18\end{array}$ & $\begin{array}{l}\text { Asthenozoospermia, oligozoospermia, teratozoospermia, } \\
\text { azoospermia, testosterone, FSH level, LH level, prolactin } \\
\text { level }\end{array}$ \\
\hline Mostafa2006 & $\begin{array}{l}\text { Amorphous sperm head, pathological sperm midpiece, } \\
\text { pathological sperm tails }\end{array}$ \\
\hline Osser1992 20 & $\begin{array}{l}\text { Amorphous sperm head, pathological sperm midpiece, } \\
\text { pathological sperm tails }\end{array}$ \\
\hline Yu2013 21 & Sperm immotility \\
\hline Zhang2013 22 & $\begin{array}{l}\text { Semen pH, sperm head defects, sperm neck defect, } \\
\text { sperm tail defect }\end{array}$ \\
\hline Zhang2015 23 & FSH level, LH level, testosterone level \\
\hline Dikshit1987 24 & Immotility of sperms and abnormal morphology \\
\hline Kunzle2003 25 & $\mathrm{pH}$, immotility of sperms and abnormal morphology \\
\hline
\end{tabular}

Abbreviations: FSH follicle stimulating hormones, $L H$ luteinizing hormone 


\section{Data extraction and review}

The search of studies was carried out with reference to the PRISMA guideline [8]. Six authors (PKB, GJ, AB, ART, MZSS and MP) independently reviewed the articles which were considered relevant to this analysis and data were extracted appropriately. The authors' names, year of publication, the study design, the endpoints which were reported, the total number of smokers and non-smokers respectively, age of patients, and the total number of events which were reported in each study were carefully extracted.

Any disagreement which was raised was spontaneously resolved by the seventh author $(\mathrm{FH})$.

With the exception of the mean age of the participants, other data at baseline were not included in this analysis for two main reasons:

- Many original studies did not include risk factors and co-morbidities at baseline;

- Baseline features which were reported in certain studies were different from those reported in other studies and a comparison would not have been possible.

\section{Statistical analysis}

The latest version of RevMan software (5.3) was used to analyze the data. This analysis involved both continuous and dichotomous data. Mean and standard deviation (SD) were used during subgroup analysis whereby pooled mean difference (MD) was calculated for the continuous data. For dichotomous data, risk ratios (RR) and $95 \%$ confidence intervals (CI) were generated to represent the results.

The statistic $Q$ test and statistic $\mathrm{I}^{2}$ test were used to evaluate heterogeneity [9]. During the subgroup analysis, statistical significance was set at a $P$ value $\leq 0.05$. A fixed effects model $\left(\mathrm{I}^{2}<50 \%\right)$ or a random effects model $\left(\mathrm{I}^{2}>\right.$ $50 \%)$ was used based upon the $\mathrm{I}^{2}$ value which was obtained during each subgroup analysis.

Each study was excluded one by one and a new analysis was carried out each time to observe any significant difference compared to the main results which were obtained (sensitivity analysis).

Ethical approval or board review approval was not required for this type of research articles.

\section{Results}

\section{Search outcomes}

Electronic search resulted in a total number of 342 articles. After a proper assessment of the titles and abstracts, we excluded 285 studies. Fifty-seven (57) full-text articles were assessed for eligibility. Among the full-text articles, further studies were eliminated because:
- They included fertile/normal male participants (8);

- They involved infertile couples without specifying the gender (3);

- They reported endpoints which were not considered relevant specifically for this analysis (9);

- They were duplicated studies (21).

Finally, 16 studies [10-25] which satisfied all the inclusion and exclusion criteria of this research were included in this analysis (Fig. 1).

\section{Basic features of the studies which were included in this analysis}

A total number of 10,823 infertile male participants (5257 smokers and 5566 non-smokers) were included in this analysis.

The main features of the original studies have been summarized in Table 2.

The study design, the participants' enrollment time periods (1985-2015), the mean age (26.5-40.5 years old), and the total number of smokers (5257 participants) and non-smokers (5566 participants) have been listed in Table 2.

Other characteristics of the participants and the reasons for exclusion have been summarized in Table 3. Majority of the patients did not consume alcohol and the minority who consumed alcohol were only moderate consumers. Participants with varicocele, cryptorchidism, aspermia, chronic diseases, genital infections, genital trauma, chromosomal abnormalities were excluded from this analysis (Table 3).

\section{Oligozoospermia and teratozoospermia}

Results of this analysis showed oligozoospermia to be significantly higher in smokers (RR: 1.29, 95\% CI: $1.05-$ $1.59 ; P=0.02)$ whereas teratozoospermia was not significantly different (RR: 1.22 , 95\% CI: 0.96-1.56; $P=0.10$ ) between the smokers and the non-smokers as illustrated in Fig. 2.

\section{Asthenozoospermia and azoospermia}

Asthenozoospermia (RR: 1.42, 95\% CI: 0.97-2.09; $P=$ 0.07 ) and azoospermia (RR: 3.02, 95\% CI: 0.23-40.01; $P$ $=0.40$ ) were not significantly different between the smokers and non-smokers (Fig. 3).

\section{Impaired motility of spermatozoa and $\mathrm{pH}$ of semen (continuous data)}

The motility of sperms was not impaired between the smokers and non-smokers (MD: 1.26, 95\% CI: [- 0.643.17]; $P=0.19)$. In addition, $\mathrm{pH}$ of semen was also similarly observed (MD: 0.04, 95\% CI: [-0.03-0.11]; $P=$ 0.30) [Fig. 4]. 


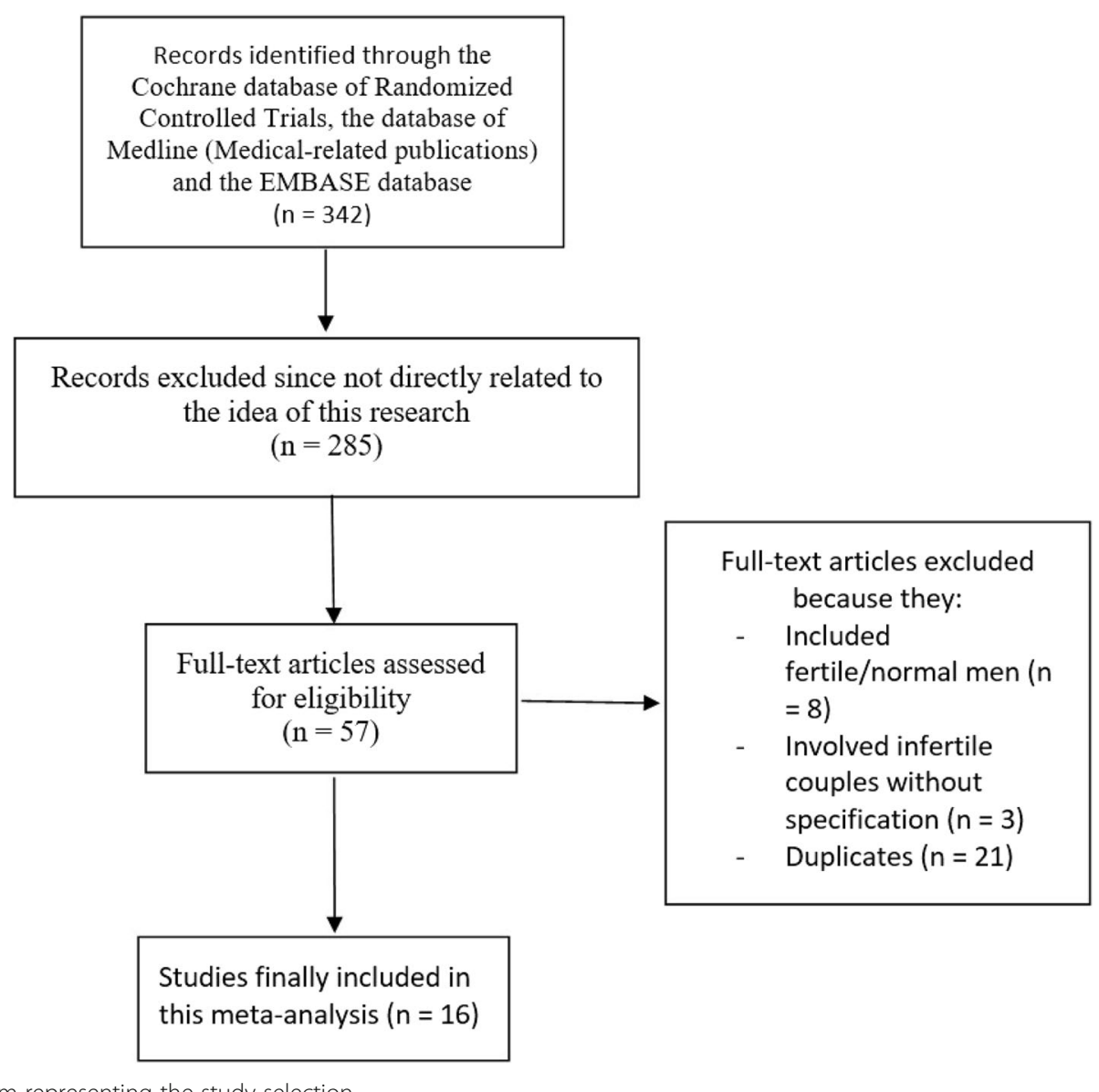

Fig. 1 Flow diagram representing the study selection

Table 2 General features of the studies

\begin{tabular}{llllll}
\hline Studies & Study design & Year of patients' enrollment & No of infertile smokers $(n)$ & No of infertile non-smokers (n) & Age (years) S/NS \\
\hline Al-Turki2014 & Retrospective & $2010-2012$ & 90 & 168 & $34.2 / 34.1$ \\
Al-Turki2016 & Retrospective & $2008-2013$ & 194 & 322 & $34.6 / 34.3$ \\
Anifandis2014 & Prospective & - & 33 & 98 & $37.9 / 37.1$ \\
Caserta2012 & Cross sectional & $2006-2011$ & 200 & 448 & $38.3 / 38.5$ \\
Cui2016 & Prospective & $2013-2015$ & 920 & 298 & - \\
Gaur2007 & Retrospective & $2001-2004$ & 100 & 100 & - \\
Meri2013 & Retrospective & $2010-2011$ & 396 & 564 & - \\
Mitra2012 & Cross sectional & - & 178 & 126 & $40.5 / 35.0$ \\
Trummer2002 & Prospective & $1993-2000$ & 478 & 517 & $31.5 / 33.4$ \\
Mostafa2006 & Prospective & - & 20 & 20 & - \\
Osser1992 & Retrospective & - & 186 & 164 & - \\
Yu2013 & Cross sectional & $2011-2012$ & 147 & 175 & $35.6 / 33.6$ \\
Zhang2013 & Retrospective & $2007-2010$ & 737 & 775 & $29.6 / 29.9$ \\
Zhang2015 & Retrospective & $2013-2014$ & 704 & 372 & $29.9 / 30.4$ \\
Dikshit1987 & Prospective & $1985-1986$ & 219 & 288 & $26.7 / 26.5$ \\
Kunzle2003 & Retrospective & $1991-1997$ & 655 & 1131 & $32.3 / 33.2$ \\
Total no of patients (n) & & 5257 & 5566 & \\
\hline Abbrevatins: 5 sm & & &
\end{tabular}


Table 3 Other characteristics and reasons for exclusion of participants

\begin{tabular}{|c|c|c|c|c|}
\hline Studies & Type of participants & Alcohol consumption & Reasons for exclusion & $\begin{array}{l}\text { Patients } \\
\text { identification }\end{array}$ \\
\hline Al-Turki2014 & $\begin{array}{l}\text { Primary and secondary } \\
\text { infertility }\end{array}$ & $\begin{array}{l}\text { Alcohol consumption was } \\
\text { controlled }\end{array}$ & Patients with azoospermia & Infertility clinic \\
\hline Al-Turki2016 & $\begin{array}{l}\text { Primary and secondary } \\
\text { infertility }\end{array}$ & $\begin{array}{l}\text { More than } 87 \% \text { of } \\
\text { participants do not } \\
\text { consume alcohol }\end{array}$ & Patients with azoospermia & Infertility clinic \\
\hline Anifandis2014 & Not specified & $\begin{array}{l}59.9 \% \text { participants do not } \\
\text { consume alcohol, and } 28 \% \\
\text { were moderate consumers }\end{array}$ & & Infertility clinic \\
\hline Caserta2012 & Primary infertility & Not specified & $\begin{array}{l}\text { Patients with azoospermia, orchitis or prostatitis, } \\
\text { grade } 2 \text { or } 3 \text { varicocele, undescended testes or its } \\
\text { surgery, altered karyotype }\end{array}$ & Infertility clinic \\
\hline Cui2016 & Primary infertility & Not specified & $\begin{array}{l}\text { Cryptorchidism, varicocele, infections, anti-sperm } \\
\text { antibodies, chromosomal abnormalities }\end{array}$ & Infertility clinic \\
\hline Gaur2007 & Primary infertility & Not specified & $\begin{array}{l}\text { Using contraceptive measures, secondary infertility, } \\
\text { occupational exposure to chemicals, cryptorchidism, } \\
\text { varicocele, chronic illness, leucocytospermia, } \\
\text { azoospermia, age }>45 \text { years }\end{array}$ & Infertility clinic \\
\hline Meri2013 & Not specified & Not specified & $\begin{array}{l}\text { Varicocele, undescended testes, small testes, } \\
\text { azoospermia, mumps, history of inguinal hernia } \\
\text { or scrotal surgery, chronic medical illness }\end{array}$ & Infertility clinic \\
\hline Mitra2012 & Not specified & Not specified & Pathology of chronic diseases & Infertility clinic \\
\hline Trummer2002 & Not specified & Not specified & Not specified & Infertility clinic \\
\hline Mostafa2006 & Not specified & Not specified & Not specified & Infertility clinic \\
\hline Osser1992 & Not specified & Not specified & Not specified & Infertility clinic \\
\hline Yu2013 & Not specified & Not specified & $\begin{array}{l}\text { Unhealthy, varicocele, infection, obstruction of } \\
\text { the vas deferens, chromosomal abnormality, } \\
\text { azoospermia, severe oligozoospermia, hemospermia, } \\
\text { leukospermia, necrozoospermia }\end{array}$ & Infertility clinic \\
\hline Zhang2013 & Not specified & Not specified & $\begin{array}{l}\text { Azoospermia, excessive alcohol intake, hallucinatory } \\
\text { drugs, serious systemic disease, abnormality of the } \\
\text { external genitalia, known family genital disorders, } \\
\text { infection or trauma to genitals }\end{array}$ & Infertility clinic \\
\hline Zhang2015 & Not specified & Not specified & Not specified & Infertility clinic \\
\hline Dikshit1987 & $\begin{array}{l}\text { Screening for idiopathic } \\
\text { infertility }\end{array}$ & No & $\begin{array}{l}\text { Past or present systemic disease, alcohol consumption, } \\
\text { genital tract disorder, varicocele, genital infection, } \\
\text { hormonal abnormalities or treatment, exposure to } \\
\text { radiation, drug abuse }\end{array}$ & Infertility clinic \\
\hline Kunzle2003 & $\begin{array}{l}\text { Men attending the andrology } \\
\text { laboratory in the context of } \\
\text { infertility investigation }\end{array}$ & Yes & $\begin{array}{l}\text { History of orchitis, testicular trauma, sexually } \\
\text { transmitted } \\
\text { disease, varicocele, inguinal hernia operation and } \\
\text { cryptorchism. }\end{array}$ & Infertility clinic \\
\hline
\end{tabular}

\section{Morphological defects of spermatozoa}

There was a significant increase in the morphological defects of spermatozoa (MD: 2.44, 95\% CI: [0.99-3.89]; $P=0.001)$ including head (MD: $1.76,95 \% \mathrm{CI}: 0.32-3.20$; $P=0.02$ ), neck (MD: 1.97, 95\% CI: 0.75-3.18; $P=0.002$ ) and tail (MD: $1.29,95 \% \mathrm{CI}: 0.35-2.22 ; P=0.007)$ defects as shown in. Figure 5.

\section{Hormones which were involved in reproduction}

This analysis did not show any significant difference in testosterone level (MD: 0.18, 95\% CI: -1.26 - 1.63; $P=0.80$ ), LH level (MD: $0.18,95 \%$ CI: -0.47 - 0.83;
$P=0.58)$ and prolactin level (MD: 1.79 , 95\% CI: -5.78 - 9.36; $P=0.64$ ) between smokers and non-smokers as shown in Fig. 6. FSH level was also not significantly decreased (MD: $0.12,95 \% \mathrm{CI}:-0.41-0.64 ; P=$ 0.66) [Fig. 7].

Table 4 has summarized the results of this analysis.

Sensitivity analysis showed that in the subgroup analyzing for teratozoospermia, excluding study Mostafa2002 showed a statistically significant result (RR: 1.32, 95\% CI: $1.03-1.70 ; P=0.03)$. Otherwise, consistent results were obtained throughout all the other subgroups. 


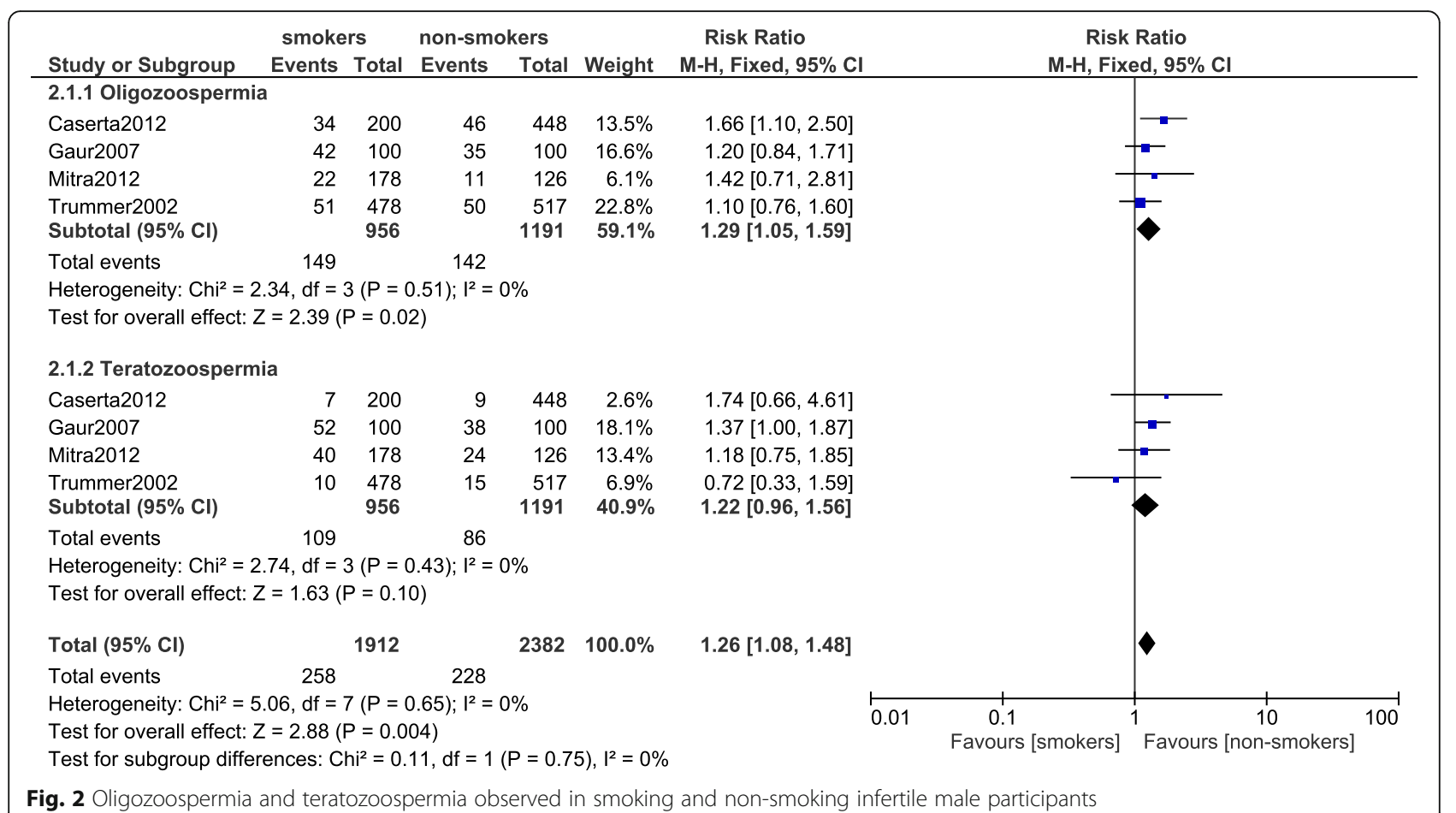

\section{Discussion}

As expected, this analysis showed smoking to have a significant impact on the quantity and quality of sperms in the infertile male participants. Tobacco smoking was associated with a lower sperm count and an increase in the number of morphological defects including head, neck and tail defects of spermatozoa. However, the $\mathrm{pH}$ and motility of spermatozoa as well as the hormones which were involved in reproduction were not affected in this population of infertile males.

A recent meta-analysis which assessed human semen showed tobacco smoking to have a negative impact on semen parameters [26]. The analysis which consisted of a total number of 5865 fertile and infertile men showed a reduced sperm count and impaired motility in semen samples of these young men. Even though the results

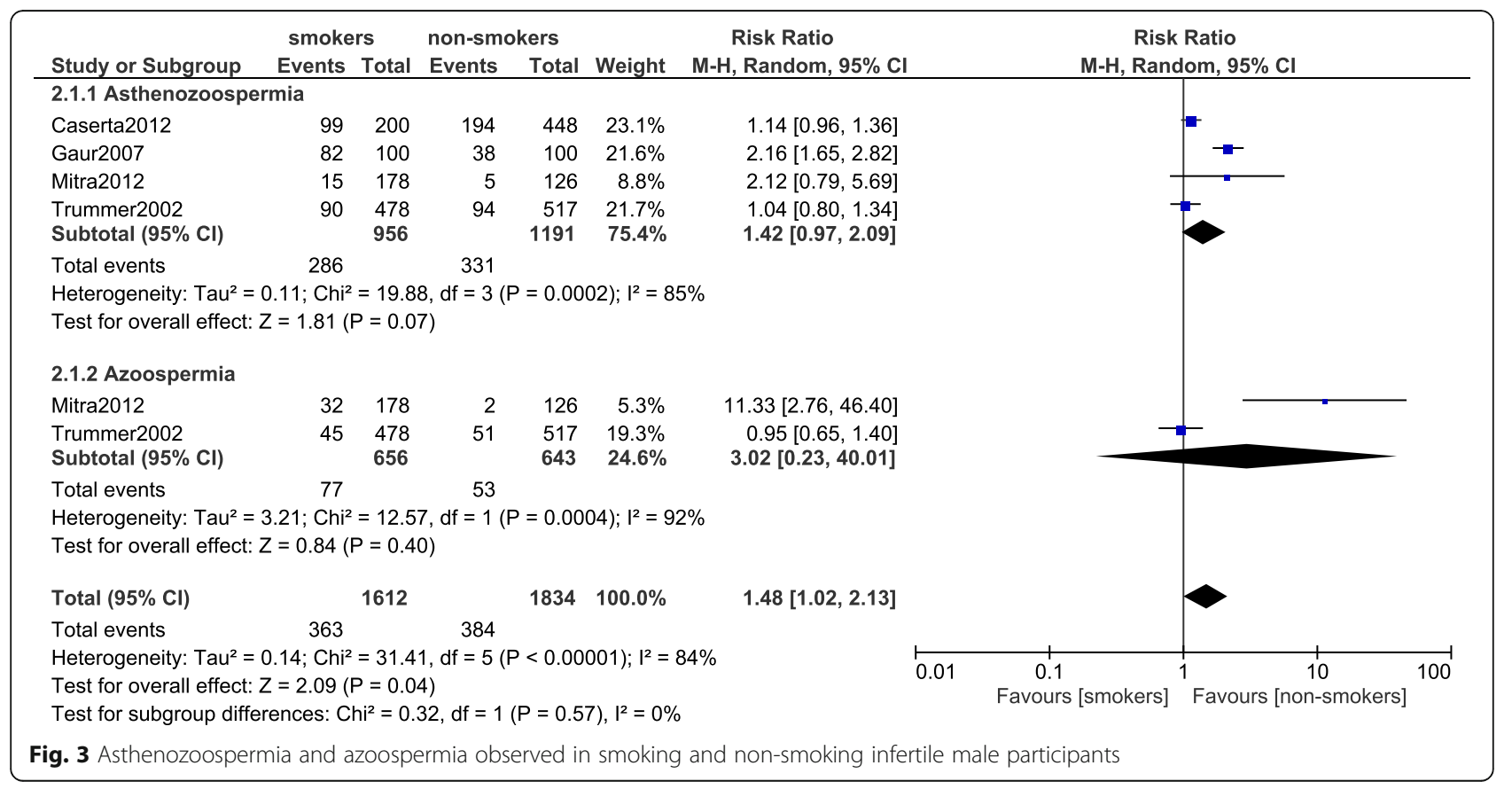




\begin{tabular}{|c|c|c|c|c|c|c|c|c|c|c|c|}
\hline \multirow[b]{2}{*}{ Study or Subgroup } & \multicolumn{3}{|c|}{ smokers } & \multicolumn{3}{|c|}{ non-smokers } & \multirow[b]{2}{*}{ Weight } & \multirow{2}{*}{$\begin{array}{l}\text { Mean Difference } \\
\text { IV, Random, } 95 \% \mathrm{Cl}\end{array}$} & \multirow{2}{*}{\multicolumn{3}{|c|}{$\begin{array}{l}\text { Mean Difference } \\
\text { IV, Random, } 95 \% \mathrm{Cl}\end{array}$}} \\
\hline & Mean & SD & Total & Mean & SD & Total & & & & & \\
\hline \multicolumn{12}{|l|}{ 1.1.1 $\mathrm{pH}$ of semen } \\
\hline Al-Turki2014 & 7.43 & 0.3 & 90 & 7.37 & 0.43 & 168 & $19.3 \%$ & $0.06[-0.03,0.15]$ & 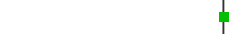 & 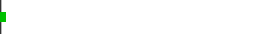 & \\
\hline Al-Turki2016 & 7.4 & 0.4 & 194 & 7.4 & 0.2 & 322 & $21.2 \%$ & $0.00[-0.06,0.06]$ & i & 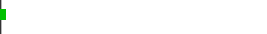 & \\
\hline Kunzle2003 & 7.5 & 0.3 & 655 & 7.4 & 0.2 & 1131 & $22.7 \%$ & $0.10[0.07,0.13]$ & i & 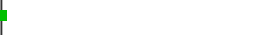 & \\
\hline $\begin{array}{l}\text { Zhang2013 } \\
\text { Subtotal (95\% Cl) }\end{array}$ & 7.12 & 0.24 & $\begin{array}{r}737 \\
1676\end{array}$ & 7.13 & 0.23 & $\begin{array}{r}775 \\
2396\end{array}$ & $\begin{array}{l}22.7 \% \\
85.9 \%\end{array}$ & $\begin{array}{c}-0.01[-0.03,0.01] \\
0.04[-0.03,0.11]\end{array}$ & 1 & 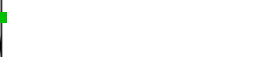 & \\
\hline \multicolumn{12}{|c|}{$\begin{array}{l}\text { Heterogeneity: } \mathrm{Tau}^{2}=0.00 ; \mathrm{Chi}^{2}=39.74, \mathrm{df}=3(P<0.00001) ; I^{2}=92 \% \\
\text { Test for overall effect: } Z=1.04(P=0.30)\end{array}$} \\
\hline \multicolumn{12}{|c|}{ 1.1.2 Immotility of sperm } \\
\hline Anifandis2014 & 35.18 & 3.6 & 33 & 33.25 & 2.1 & 98 & $0.6 \%$ & $1.93[0.63,3.23]$ & & $=$ & \\
\hline Dikshit1987 & 39.41 & 1.04 & 219 & 39.98 & 0.95 & 288 & $13.2 \%$ & $-0.57[-0.75,-0.39]$ & & 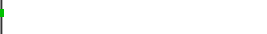 & \\
\hline Kunzle2003 & 62.9 & 18.6 & 655 & 61.3 & 17.7 & 1131 & $0.3 \%$ & $1.60[-0.16,3.36]$ & & $=$ & \\
\hline Yu2013 & 55 & 20 & 147 & 51 & 20 & 175 & $0.0 \%$ & $4.00[-0.39,8.39]$ & & F & \\
\hline \multicolumn{12}{|c|}{$\begin{array}{l}\text { Heterogeneity: } \mathrm{Tau}^{2}=2.83 ; \mathrm{Chi}^{2}=23.62, \mathrm{df}=3(\mathrm{P}<0.0001) ;\left.\right|^{2}=87 \% \\
\text { Test for overall effect: } Z=1.30(P=0.19)\end{array}$} \\
\hline Total $(95 \% \mathrm{Cl})$ & & & 2730 & & & 4088 & $100.0 \%$ & $-0.03[-0.12,0.07]$ & & & \\
\hline \multicolumn{8}{|c|}{$\begin{array}{l}\text { Heterogeneity: } \mathrm{Tau}^{2}=0.01 ; \mathrm{Chi}^{2}=99.56, \mathrm{df}=7(P<0.00001) ; \mathrm{I}^{2}=93 \% \\
\text { Test for overall effect: } Z=0.51(\mathrm{P}=0.61) \\
\text { Test for subgroup differences: } \mathrm{Chi}^{2}=1.59, \mathrm{df}=1(\mathrm{P}=0.21), \mathrm{I}^{2}=37.2 \%\end{array}$} & -100 & $\begin{array}{c}-50 \\
\text { Favours [smokers] }\end{array}$ & $\begin{array}{c}1 \\
\text { Favours [non-smokers] }\end{array}$ & $\overrightarrow{100}$ \\
\hline
\end{tabular}

\begin{tabular}{|c|c|c|c|c|c|c|c|c|c|c|c|}
\hline \multirow[b]{2}{*}{ Study or Subgroup } & \multicolumn{3}{|c|}{ smokers } & \multicolumn{3}{|c|}{ non-smokers } & \multirow[b]{2}{*}{ Weight } & \multirow{2}{*}{$\begin{array}{l}\text { Mean Difference } \\
\text { IV, Random, } 95 \% \mathrm{CI}\end{array}$} & \multirow{2}{*}{\multicolumn{3}{|c|}{$\begin{array}{l}\text { Mean Difference } \\
\text { IV, Random, } 95 \% \mathrm{Cl}\end{array}$}} \\
\hline & Mean & SD & Total & Mean & SD & Total & & & & & \\
\hline \multicolumn{12}{|c|}{ 1.2.1 Abnormal forms of sperm } \\
\hline Dikshit1987 & 20.24 & 0.81 & 219 & 19.35 & 0.57 & 288 & $10.4 \%$ & $0.89[0.76,1.02]$ & & 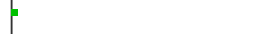 & \\
\hline Kunzle2003 & 78.8 & 14.6 & 655 & 76.3 & 15.5 & 1131 & $3.2 \%$ & $2.50[1.06,3.94]$ & & $=$ & \\
\hline Meri2013 & 73 & 11.7 & 396 & 60.7 & 11.7 & 564 & $3.0 \%$ & $12.30[10.80,13.80]$ & & $=$ & \\
\hline Mostafa2006 & 14.4 & 3.59 & 20 & 13.5 & 3.28 & 20 & $1.7 \%$ & $0.90[-1.23,3.03]$ & & + & \\
\hline Osser1992 & 49.6 & 1.1 & 186 & 49.9 & 1.2 & 164 & $10.0 \%$ & $-0.30[-0.54,-0.06]$ & & 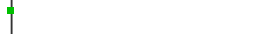 & \\
\hline $\begin{array}{l}\text { Trummer2002 } \\
\text { Subtotal }(95 \% \mathrm{CI})\end{array}$ & 56.1 & 17.9 & $\begin{array}{r}478 \\
1954\end{array}$ & 57.8 & 17.6 & $\begin{array}{r}517 \\
2684\end{array}$ & $\begin{array}{r}1.6 \% \\
29.9 \%\end{array}$ & $-1.70[-3.91,0.51]$ & & 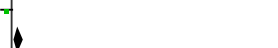 & \\
\hline \multicolumn{12}{|c|}{$\begin{array}{l}\text { Heterogeneity: } \mathrm{Tau}^{2}=2.76 ; \mathrm{Chi}^{2}=313.45, \mathrm{df}=5(P<0.00001) ;\left.\right|^{2}=98 \% \\
\text { Test for overall effect: } Z=3.29(P=0.0010)\end{array}$} \\
\hline \multicolumn{12}{|l|}{ 1.2.2 Head defects } \\
\hline Cui2016 & 88.38 & 15.11 & 920 & 82.5 & 11.66 & 298 & $2.6 \%$ & $5.88[4.24,7.52]$ & & $=$ & \\
\hline Osser1992 & 47.2 & 1 & 186 & 47 & 1.2 & 164 & $10.0 \%$ & $0.20[-0.03,0.43]$ & & t & \\
\hline $\begin{array}{l}\text { Zhang2013 } \\
\text { Subtotal (95\% Cl) }\end{array}$ & 88.32 & 4.3 & $\begin{array}{r}562 \\
1668\end{array}$ & 87.81 & 3.78 & $\begin{array}{r}615 \\
1077\end{array}$ & $\begin{array}{r}8.5 \% \\
21.2 \%\end{array}$ & $\begin{array}{c}0.51[0.05,0.97] \\
1.76[0.32,3.20]\end{array}$ & & 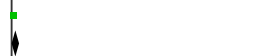 & \\
\hline \multicolumn{12}{|c|}{$\begin{array}{l}\text { Heterogeneity: } \mathrm{Tau}^{2}=1.41 ; \mathrm{Chi}^{2}=45.46, \mathrm{df}=2(\mathrm{P}<0.00001) ; \mathrm{I}^{2}=96 \% \\
\text { Test for overall effect: } Z=2.40(P=0.02)\end{array}$} \\
\hline \multicolumn{12}{|l|}{ 1.2.3 Neck defect } \\
\hline Cui2016 & 49.32 & 14.43 & 920 & 41.38 & 8.58 & 298 & $3.5 \%$ & $7.94[6.59,9.29]$ & & $=$ & \\
\hline Osser1992 & 1.8 & 0.1 & 186 & 1.8 & 0.1 & 164 & $10.6 \%$ & $0.00[-0.02,0.02]$ & & $\phi$ & \\
\hline Zhang2013 & 2.15 & 2.02 & 562 & 2.34 & 2.29 & $\begin{array}{r}615 \\
1077\end{array}$ & $9.9 \%$ & $-0.19[-0.44,0.06]$ & & 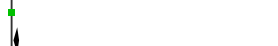 & \\
\hline \multicolumn{12}{|c|}{$\begin{array}{l}\text { Heterogeneity: } \mathrm{Tau}^{2}=1.03 ; \mathrm{Chi}^{2}=135.47, \mathrm{df}=2(P<0.00001) ;\left.\right|^{2}=99 \% \\
\text { Test for overall effect: } Z=3.17(P=0.002)\end{array}$} \\
\hline \multicolumn{12}{|l|}{ 1.2.4 Tail defect } \\
\hline Cui2016 & 11.64 & 12.77 & 920 & 6.23 & 7.19 & 298 & $4.2 \%$ & $5.41[4.25,6.57]$ & & $=$ & \\
\hline Osser1992 & 3 & 0.2 & 186 & 3.1 & 0.3 & 164 & $10.6 \%$ & $-0.10[-0.15,-0.05]$ & & $\phi$ & \\
\hline $\begin{array}{l}\text { Zhang2013 } \\
\text { Subtotal (95\% Cl) }\end{array}$ & 2.3 & 2.21 & $\begin{array}{r}562 \\
1668\end{array}$ & 2.24 & 1.71 & $\begin{array}{r}615 \\
1077\end{array}$ & $\begin{array}{l}10.0 \% \\
24.8 \%\end{array}$ & $\begin{array}{l}0.06[-0.17,0.29] \\
1.29[0.35,2.22]\end{array}$ & & 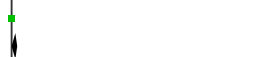 & \\
\hline \multicolumn{12}{|c|}{$\begin{array}{l}\text { Heterogeneity: } \text { Tau}^{2}=0.59 ; \mathrm{Chi}^{2}=87.92, \mathrm{df}=2(P<0.00001) ; I^{2}=98 \% \\
\text { Test for overall effect: } Z=2.71(P=0.007)\end{array}$} \\
\hline Total $(95 \% \mathrm{Cl})$ & & & 6958 & & & 5915 & $100.0 \%$ & $1.20[0.89,1.50]$ & & & \\
\hline \multicolumn{9}{|c|}{$\begin{array}{l}\text { Heterogeneity: } \mathrm{Tau}^{2}=0.23 ; \mathrm{Chi}^{2}=756.45, \mathrm{df}=14(P<0.00001) ; \mathrm{I}^{2}= \\
\text { Test for overall effect: } Z=7.65(P<0.00001) \\
\text { Test for subgroup differences: } \mathrm{Chi}^{2}=1.93, \mathrm{df}=3(\mathrm{P}=0.59), \mathrm{I}^{2}=0 \%\end{array}$} & $\begin{array}{l}-50 \\
\text { Favours [smokers] }\end{array}$ & ${ }^{0}$ Favours [non-smokers] & 100 \\
\hline
\end{tabular}




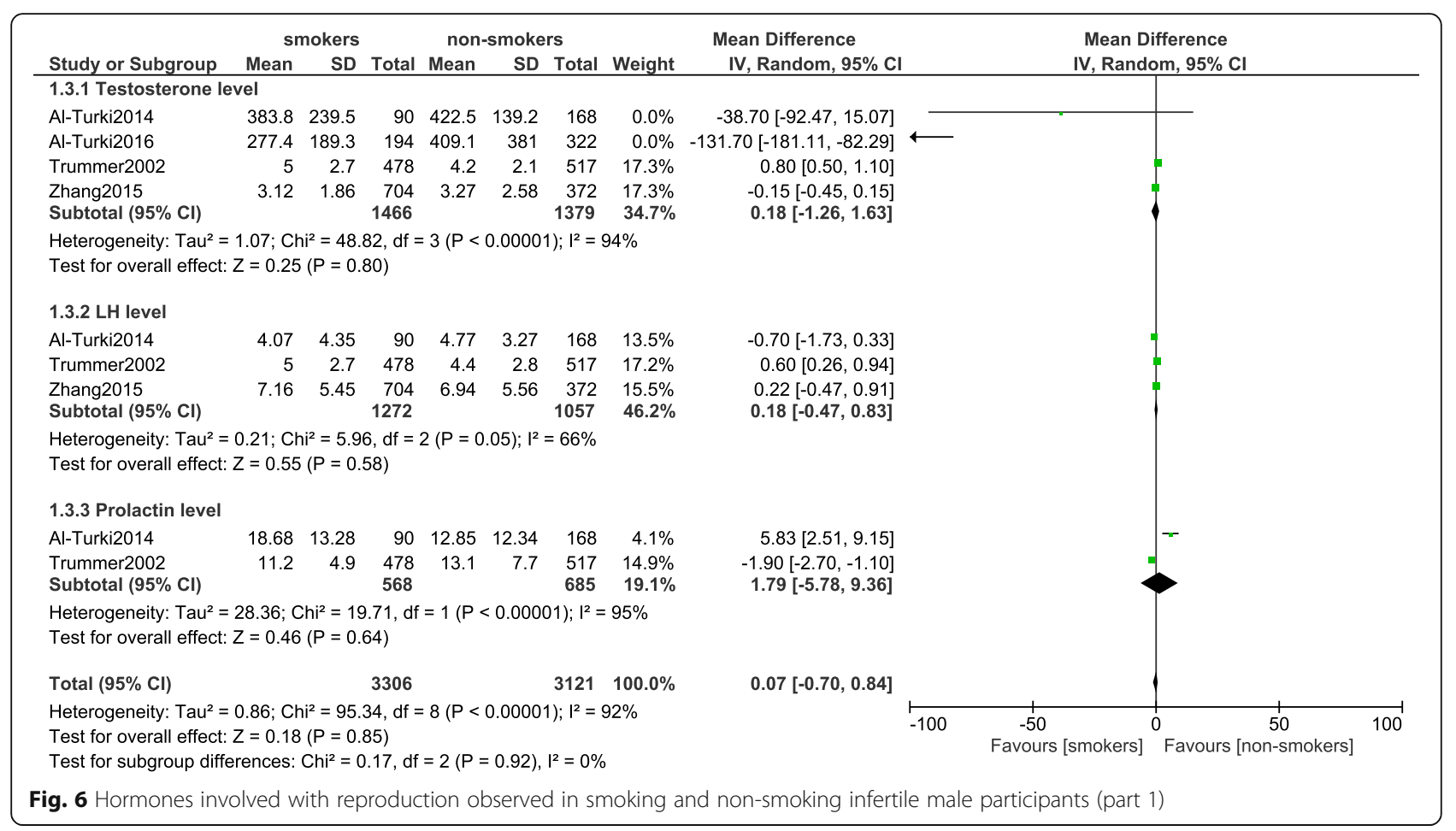

which were obtained were almost similar with respect to this current analysis, the other analysis included only studies which were published between the years 2010 to 2015, whereas our current analysis included studies which were published even before the year 2010. Another difference with respect to the current analysis was the fact that there was no language barrier in the other analysis. Moreover, the other analysis also assessed results with reference to the total number of cigarettes which were consumed daily. In contrast to the other analysis, this current meta-analysis assessed specific morphological defects, as well as any dis-balance of the hormones which were involved in reproduction.

Another study evaluating the effect of cigarette smoking on vital seminal parameters which influence fertility showed smoking to cause impaired motility to a higher extent in comparison to the impairment in sperm count [27]. Men with primary infertility aged between 25 to 40 years were included and a follow-up period of less and above 5 years were considered.

A case control study also showed smoking to be associated with a lower semen concentration, impaired motility of spermatozoa and an increased morphology defect [28] in part reflecting the results of this current analysis. Additionally, an article published by the Canadian Society of Clinical Chemists showed that abnormal structural defects of spermatozoa, especially round head defects, were associated with tobacco smoking which might be attributed to increased oxidative stress and insufficient scavenging antioxidant enzymes in the seminal fluids of infertile men [29]. Other studies have shown zinc to contribute to this unwanted mechanism in

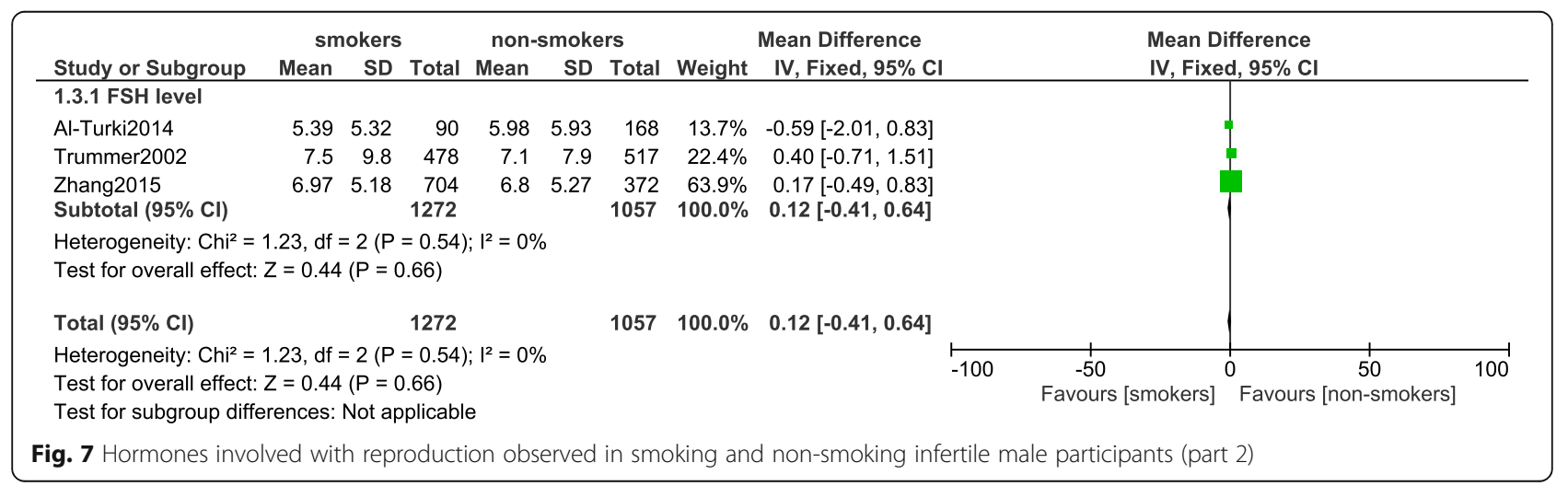


Table 4 Results of this analysis

\begin{tabular}{|c|c|c|c|c|}
\hline Endpoints & No of studies involved $(n)$ & RR or MD with $95 \% \mathrm{Cl}$ & $P$ value & $P^{2}(\%)$ \\
\hline Oligozoospermia & 4 & $1.29[1.05-1.59]$ & 0.02 & 0 \\
\hline Teratozoospermia & 3 & $1.22[0.96-1.56]$ & 0.10 & 0 \\
\hline Asthenozoospermia & 4 & $1.42[0.97-2.09]$ & 0.07 & 85 \\
\hline Azoospermia & 2 & $3.02[0.23-40.01]$ & 0.40 & 92 \\
\hline $\mathrm{pH}$ of semen & 4 & $0.04[-0.03-0.11]$ & 0.30 & 92 \\
\hline Impaired motility of sperm (continuous data) & 4 & $1.26[-0.64-3.17]$ & 0.19 & 87 \\
\hline Abnormal form of sperm & 6 & $2.44[0.99-3.89]$ & 0.001 & 98 \\
\hline Head defects & 3 & $1.76[0.32-3.20]$ & 0.02 & 96 \\
\hline Neck defects & 3 & $1.97[0.75-3.18]$ & 0.002 & 99 \\
\hline Tail defects & 3 & $1.29[0.35-2.22]$ & 0.007 & 98 \\
\hline Testosterone level & 4 & $0.18[-1.26-1.63]$ & 0.80 & 94 \\
\hline LH level & 3 & $0.18[-0.47-0.83]$ & 0.58 & 66 \\
\hline Prolactin level & 2 & $1.79[-5.78-9.36]$ & 0.64 & 95 \\
\hline FSH level & 3 & $0.12[-0.41-0.64]$ & 0.66 & 0 \\
\hline
\end{tabular}

Abbreviations: $M D$ mean difference, $R R$ risk ratio, $C l$ confidence intervals, $L H$ luteinizing hormone, $F S H$ follicle stimulating hormone

infertile smokers [30]. Other mechanisms have well been explained in previously published reviews [31,32].

Briefly, the possible mechanisms which might be involved with the effect of cigarette smoking on semen parameters are: toxic contents found in cigarette smoking might have harmful effects on male germ cells and their developmental processes [33]. Negative effects of nicotine on semen parameters have also previously been reported [34]. Other possible mechanisms might be related to the negative impact of smoking on the 8 nAChR subunits found in human spermatozoa, resulting in smoking-related sperm damage [35]. In addition, different proteins (Aldoa, ATP5a1, Gpx4, Cs) expressed in sperms were significantly altered in smokers [36]. Cigarette smoking was found to also affect $\mathrm{Ca}^{2+}$ - ATPase activity of the spermatozoa as well [37].

However, even though clinical research has shown smoking to have an adverse effect on the progressive sperm motility irrespective of the total number of cigarettes smoked daily [38, 39], other studies showed no relationship between smoking and male infertility [40].

This current analysis showed no significant influence of smoking on testosterone, prolactin, FSH and LH levels. To support this point, Wang et al. showed smoking not to be an independent predictor of sex-hormone binding globulin even though a relation or linked was observed between increasing packets of cigarette and sex-hormone binding globulin [41]. Similarly, another study conducted in Taiwan showed no significant difference in LH and FSH levels between smokers and non-smokers [42] showing smoking to have a much higher impact on semen compared to the production of hormones which were involved in the functioning of the male reproductive system.
Several alternative methods to stop smoking have been suggested [43-46]. However, apart from smoking, other factors such as regular heavy alcohol consumption [47], certain medications, co-morbidities, autoimmune diseases and other environmental factors might also contribute to abnormalities in semen parameters, morphology and impaired motility and should further be investigated [48].

This interesting research should inspire other scientists to investigate more about the mechanisms, the factors associated with a poor semen quality in smokers; in order for proper actions to be taken in a timely manner to reduce this serious dilemma faced by several young men and couples in our society.

This meta-analysis should be considered new for the following reasons: it is among the only few meta-analyses to systematically show the impact of smoking on the quality of semen in infertile males. This article might be considered new on the basis of the total number of participants and the number of different endpoints which were analyzed in one particular study.

\section{Limitations}

Limitations might be the fact that a high level of heterogeneity was observed among several of the subgroups analyzing the different endpoints. This could be due to the inclusion of observational data. In addition, several endpoints were analyzed only using a small number of studies. Factors such as alcohol consumption could have had an influence on the main results. Moreover, the infertility duration, and other associated factors such as genital infections, varicocele, environmental factors were not clearly reported in several studies. 


\section{Conclusions}

In conclusion, with reference to the clinical endpoints which were studied in this analysis, tobacco smoking was associated with a lower sperm count and an increase in the number of morphological defects of spermatozoa. However, the $\mathrm{pH}$ and motility of spermatozoa as well as the production of hormones which were involved in reproduction were not affected in this population of infertile males.

\section{Abbreviations}

Cl: confidence intervals; FSH: follicle stimulating hormones; LH: luteinizing hormone; RR: risk ratios

\section{Acknowledgements}

Not applicable.

\section{Funding}

No external funding was used in the preparation of this manuscript. This research was supported by National Natural Science Foundation of China (No. 81560046, 81760057) and Guangxi Natural Science Foundation (No. 2016GXNSFAA380002)

\section{Availability of data and materials}

All data and materials used in this research are freely available. References have been provided.

\section{Authors' contributions}

PKB, GJ, MZSS, AB, ART, MP and FH were responsible for the conception and design, acquisition of data, analysis and interpretation of data, drafting the initial manuscript and revising it critically for important intellectual content. PKB wrote this manuscript. All authors read and approved the final manuscript as presented.

\section{Authors' information}

Dr. Pravesh Kumar Bundhun (M.D) is the first author. From the Department of Internal Medicine, the First Affiliated Hospital of Guangxi Medical University, Nanning, Guangxi, China.

\section{Ethics approval and consent to participate}

Ethical approval was not applicable for this systematic review and metaanalysis.

\section{Consent for publication}

Not applicable.

\section{Competing interests}

The authors declare that they have no competing interests.

\section{Publisher's Note}

Springer Nature remains neutral with regard to jurisdictional claims in published maps and institutional affiliations.

\section{Author details}

${ }^{1}$ Department of Internal Medicine, the First Affiliated Hospital of Guangxi Medical University, Nanning, Guangxi 530021, People's Republic of China. ${ }^{2}$ Guangxi Medical University, Nanning, Guangxi 530027, People's Republic of China. ${ }^{3}$ Institute of Cardiovascular Diseases and Guangxi Key Laboratory Base of Precision Medicine in Cardio-cerebrovascular Disease Control and Prevention and Guangxi Clinical Research Center for Cardio-cerebrovascular Diseases, the First Affiliated Hospital of Guangxi Medical University, Nanning 530021, Guangxi, China.
Received: 31 March 2018 Accepted: 12 December 2018

Published online: 08 January 2019

\section{References}

1. Corrao MA, Guindon GE, Cokkinides V, Sharma N. Building the evidence base for global tobacco control. Bull World Health Organ. 2000;78(7):884-90.

2. Rojewski AM, Zuromski KL, Toll BA. Strategies for smoking cessation among high risk populations to prevent lung cancer. Expert Rev Respir Med. 2016 Dec 13:1-3.

3. Xu X, Chen C, Abdullah AS, Sharma M, Liu H, Zhao Y. Knowledge about and sources of smoking-related knowledge, and influencing factors among male urban secondary school students in Chongqing. China Springerplus. 2016:5(1):1879.

4. Patel A, Sharma PS, Narayan P, Nair BV, Narayanakurup D, Pai PJ. Distress in infertile males in Manipal-India: a clinic based study. J Reprod Infertil. 2016; 17(4):213-20.

5. Practice Committee of American Society for Reproductive Medicine Definitions of infertility and recurrent pregnancy loss: a committee opinion. Fertil Steril. 2013;99(1):63

6. Daumler D, Chan P, Lo KC, Takefman J, Zelkowitz P. Men's knowledge of their own fertility: a population-based survey examining the awareness of factors that are associated with male infertility. Hum Reprod. 2016;31(12): 2781-90.

7. Kovac JR, Pastuszak AW, Lamb DJ. The use of genomics, proteomics, and metabolomics in identifying biomarkers of male infertility. Fertil Steril. 2013; 99(4):998-1007.

8. Liberati A, Altman DG, Tetzlaff J, et al. The PRISMA statement for reporting systematic reviews and meta-analyses of studies that evaluate healthcareinterventions: explanation and elaboration. BMJ. 2009;339:b2700.

9. Julian P T Higgins, Statistician, Simon G Thompson, director, Jonathan J Deeks, senior medical statistician, and, Douglas G Altman, professor of statistics in medicine. Measuring inconsistency in meta-analyses. BMJ 2003 Sep 6: 327(7414): 557-560.

10. Al-Turki HA. Effect of smoking on reproductive hormones and semen parameters of infertile Saudi Arabians. Urol Ann. 2015;7(1):63-6.

11. Al-Turki HA. A 5-year analysis of semen parameters in Saudi Arabian men attending infertility clinics. J Int Med Res. 2016:44(3):656-61.

12. Anifandis G, Bounartzi T, Messini Cl, Dafopoulos K, Sotiriou S, Messinis IE. The impact of cigarette smoking and alcohol consumption on sperm parameters and sperm DNAfragmentation (SDF) measured by Halosperm ( ${ }^{\circledR}$. Arch Gynecol Obstet. 2014;290(4):777-82.

13. Caserta D, Bordi G, Di Segni N, D'Ambrosio A, Mallozzi M, Moscarini M. The influence of cigarette smoking on a population of infertile men and women. Arch Gynecol Obstet. 2013;287(4):813-8.

14. Cui $X$, Jing $X$, Wu $X$, Wang Z, Li Q. Potential effect of smoking on semen quality through DNA damage and the downregulation of Chk1 in sperm. Mol Med Rep. 2016;14(1):753-61.

15. Gaur DS, Talekar M, Pathak VP. Effect of cigarette smoking on semen quality of infertile men. Singap Med J. 2007:48(2):119-23.

16. Meri ZB, Irshid IB, Migdadi M, Irshid AB, Mhanna SA. Does cigarette smoking affect seminal fluid parameters? A comparative study. Oman Med J. 2013; 28(1):12-5.

17. Mitra A, Chakraborty B, Mukhopadhay D, Pal M, Mukherjee S, Banerjee S, Chaudhuri K. Effect of smoking on semen quality, FSH, testosterone level, and CAG repeat length in androgen receptorgene of infertile men in an Indian city. Syst Biol Reprod Med. 2012;58(5):255-62.

18. Trummer $\mathrm{H}$, Habermann $\mathrm{H}$, Haas J, Pummer $\mathrm{K}$. The impact of cigarette smoking on human semen parameters and hormones. Hum Reprod. 2002;17(6):1554-9.

19. Mostafa T, Tawadrous G, Roaia MM, Amer MK, Kader RA, Aziz A. Effect of smoking on seminal plasma ascorbic acid in infertile and fertile males. Andrologia. 2006:38(6):221-4

20. Osser S, Beckman-Ramirez A, Liedholm P. Semen quality of smoking and non-smoking men in infertile couples in a Swedish population. Acta Obstet Gynecol Scand. 1992;71(3):215-8.

21. Yu B, Qi Y, Liu D, Gao X, Chen H, Bai C, Huang Z. Cigarette smoking is associated with abnormal histone-to-protamine transition in human sperm. Fertil Steril. 2014;101(1):51-57.e1.

22. Zhang ZH, Zhu HB, Li LL, Yu Y, Zhang HG, Liu RZ. Decline of semen quality and increase of leukocytes with cigarette smoking in infertile men. Iran J Reprod Med. 2013:11(7):589-96.

23. Zhang M, Zhang QS, Zheng HS, Wang XY, Feng SQ, Tian WJ, Fan HT. Clinical, demographic and psychological characteristics of infertile male smokers in Northeast China. J Int Med Res. 2016;44(1):75-80. 
24. Dikshit RK, Buch JG, Mansuri SM. Effect of tobacco consumption on semen quality of a population of hypofertile males. Fertil Steril. 1987;48(2):334-6.

25. R K, Mueller MD, Hänggi W, Birkhäuser $M H$, Drescher $H$, Bersinger NA. Semen quality of male smokers and nonsmokers in infertile couples. Fertil Steril. 2003;79(2):287-91.

26. Sharma R, Harlev A, Agarwal A, Esteves SC. Cigarette smoking and semen quality: a new meta-analysis examining the effect of the 2010 World Health Organization Laboratory methods for the examination of human semen. Eur Urol. 2016;70(4):635-45.

27. Lingappa HA, Govindashetty AM, Puttaveerachary AK, Manchaiah S, Krishnamurthy A, Bashir S, Doddaiah N. Evaluation of effect of cigarette smoking on vital seminal parameters which influence fertility. J Clin Diagn Res. 2015;9(7):EC13-5.

28. Davar R, Sekhavat $L$, Naserzadeh N. Semen parameters of non-infertile smoker and non-smoker men. J Med Life. 2012;5(4):465-8.

29. Elshal MF, El-Sayed IH, Elsaied MA, El-Masry SA, Kumosani TA. Sperm head defects and disturbances in spermatozoal chromatin and DNA integrities in idiopathicinfertile subjects: association with cigarette smoking. Clin Biochem. 2009;42(7-8):589-94.

30. Liu RZ, Gao JC, Zhang HG, Wang RX, Zhang ZH, Liu XY. Seminal plasma zinc level may be associated with the effect of cigarette smoking on sperm parameters. J Int Med Res. 2010;38(3):923-8.

31. Mak V, Jarvi K, Buckspan M, Freeman M, Hechter S, Zini A. Smoking is associated with the retention of cytoplasm by human spermatozoa. Urology. 2000;56(3):463-6.

32. Harlev A, Agarwal A, Gunes SO, Shetty A, du Plessis SS. Smoking and male infertility: an evidence-based review. World J Mens Health. 2015;33(3):143-60.

33. Zenzes MT. Smoking and reproduction: gene damage to human gametes and embryos. Hum Reprod Update. 2000;6(2):122-31.

34. Pacifici R, Altieri I, Gandini L, Lenzi A, Pichini S, Rosa M, Zuccaro P, Dondero F. Nicotine, cotinine, and trans-3-hydroxycotinine levels in seminal plasma of smokers: effects on sperm parameters. Ther Drug Monit. 1993;15(5):358-63.

35. Condorelli RA, La Vignera S, Duca Y, Zanghi GN, Calogero AE. Nicotine receptors as a possible marker for smoking-related sperm damage. Protein Pept Lett. 2018;25(5):451-4.

36. Chen X, Xu W, Miao M, Zhu Z, Dai J, Chen Z, Fang P, Wu J, Nie D, Wang L, Wang Z, Qiao Z, Shi H. Alteration of sperm protein profile induced by cigarette smoking. Acta Biochim Biophys Sin Shanghai. 2015:47(7):504-15.

37. Kumosani TA, Elshal MF, Al-Jonaid AA, Abduljabar HS. The influence of smoking on semen quality, seminal microelements and Ca2+-ATPase activity among infertile and fertile men. Clin Biochem. 2008;41(14-15):1199-203.

38. Colagar AH, Jorsaraee GA, Marzony ET. Cigarette smoking and the risk of male infertility. Pak J Biol Sci. 2007 Nov 1;10(21):3870-4.

39. Hassa H, Yildirim A, Can C, Turgut M, Tanir HM, Senses T, Sahinmutlu F. Effect of smoking on semen parameters of men attending an infertility clinic. Clin Exp Obstet Gynecol. 2006;33(1):19-22.

40. Dunphy BC, Barratt CL, von Tongelen BP, Cooke ID. Male cigarette smoking and fecundity in couples attending an infertility clinic. Andrologia. 1991; 23(3):223-5.

41. Wang W, Yang X, Liang J, M L, H Z, X Q, L M, W L, Z M. Cigarette smoking has a positive and independent effect on testosterone levels. Hormones (Athens). 2013;12(4):567-77.

42. Jeng HA, Chen YL, Kantaria KN. Association of cigarette smoking with reproductive hormone levels and semen quality in healthy adult men in Taiwan. J Environ Sci Health A Tox Hazard Subst Environ Eng. 2014;49(3):262-8.

43. Kazemzadeh Z, Manzari ZS, Pouresmail Z. Nursing interventions for smoking cessation in hospitalized patients: a systematic review. Int Nurs Rev. 2016.

44. Wang TW, Kenemer B, Tynan MA, Singh T, King B. Consumption of combustible and smokeless tobacco - United States, 2000-2015. MMWR Morb Mortal Wkly Rep. 2016 Dec 9;65(48):1357-63.

45. Sozańska B, Pearce N, Błaszczyk M, A B, Cullinan P. Changes in the prevalence of cigarette smoking and quitting smoking determinants in adult inhabitants of rural areas in Poland between 2003 and 2012. Public Health. 2016 Dec;141:178-84.

46. Lopez E. The efficacy of smoking cessation interventions via social media. Am J Public Health. 2017;107(1):e9.

47. Keskin MZ, Budak S, Gubari S, Durmaz K, Yoldas M, Celik O, Aksoy EE, Ilbey YO. Do cigarette and alcohol affect semen analysis? Arch Ital Urol Androl. 2016;88(1):56-9.

48. Gerhard I, Lenhard K, Eggert-Kruse W, Runnebaum B. Clinical data which influence semen parameters in infertile men. Hum Reprod. 1992;7(6):830-7.

Ready to submit your research? Choose BMC and benefit from:

- fast, convenient online submission

- thorough peer review by experienced researchers in your field

- rapid publication on acceptance

- support for research data, including large and complex data types

- gold Open Access which fosters wider collaboration and increased citations

- maximum visibility for your research: over $100 \mathrm{M}$ website views per year

At $\mathrm{BMC}$, research is always in progress.

Learn more biomedcentral.com/submissions 